\title{
Editorial: Recent Advances and Applications of Seismic Isolation and Energy Dissipation Devices
}

\author{
Dario De Domenico ${ }^{1 *}$, Enrico Tubaldi ${ }^{2}$, Izuru Takewaki ${ }^{3}$, Theodore Karavasilis ${ }^{4}$, \\ Andrea Dall'Asta ${ }^{5}$ and Oren Lavan ${ }^{6}$ \\ ${ }^{1}$ Department of Engineering, University of Messina, Messina, Italy, ${ }^{2}$ Department of Civil and Environmental Engineering, \\ University of Strathclyde, Glasgow, United Kingdom, ${ }^{3}$ Department of Architecture and Architectural Engineering, Kyoto \\ University, Kyoto, Japan, ${ }^{4}$ Department of Civil Engineering, University of Patras, Patras, Greece, ${ }^{5}$ School of Architecture and \\ Design, University of Camerino, Ascoli Piceno, Italy, ${ }^{6}$ Faculty of Civil and Environmental Engineering, Technion-Israel Institute \\ of Technology, Haifa, Israel
}

Keywords: seismic base isolation, energy dissipation devices, tuned mass damper, performance-based seismic engineering, reliability-based design, dampers, damper optimization, earthquake-resistant structures

\section{Editorial on the Research Topic}

\section{Recent Advances and Applications of Seismic Isolation and Energy Dissipation Devices}

One of the major (and oldest) challenges in the earthquake engineering field has been and still continues to be the conceptualization, development, and implementation of innovative earthquake-resistant systems for reducing the vulnerability of structures and infrastructures and improving the seismic performance and resilience, while keeping construction costs reasonable.

Edited by:

Massimo Latour,

University of Salerno, Italy

Reviewed by:

Carmine Galasso,

University College London,

United Kingdom

*Correspondence:

Dario De Domenico

dario.dedomenico@unime.it

Specialty section:

This article was submitted to

Earthquake Engineering,

a section of the journal

Frontiers in Built Environment

Received: 29 June 2020

Accepted: 10 July 2020

Published: 18 August 2020

Citation:

De Domenico D, Tubaldi E, Takewaki I, Karavasilis T, Dall'Asta A and Lavan O (2020) Editorial: Recent Advances and Applications of Seismic Isolation and Energy Dissipation Devices.

Front. Built Environ. 6:126. doi: 10.3389/fbuil.2020.00126
There is no doubt that seismic isolation and energy dissipation devices belong to such class of systems. The effectiveness of these technologies in protecting structural elements and non-structural components under seismic action has been proven by many theoretical and numerical studies in the literature, shake-table test results, as well as by experimental evidence on how they actually behaved during real earthquakes. A traditional earthquake-resistant design philosophy is mainly focused on the "life-safety" performance level, which implies that the structure undergoes significant damage but does not collapse during a major earthquake, so that the occupants can evacuate safely. This is certainly adequate (and somehow reasonable from economic perspectives) for ordinary structures. In contrast, a design strategy using energy dissipation devices and/or seismic isolation aims at a more challenging "functionality" performance level even under strong earthquakes. This is accomplished in a twofold manner: (1) by supplemental damping mechanisms engaged in a limited number of elements or "fuse components," which can be easily replaced or whose accumulated plastic deformations can be recovered after the earthquake; (2) by limiting the transmission of seismic energy via low lateral stiffness devices interposed between the main structure and the ground. Overall, both these strategies result in a low-damage structural system, wherein the structure can be designed to remain in an elastic or, at least, in a quasi-elastic range of the response. Until some years ago, this "high-performance level" design was deemed necessary for strategic structures requiring minimal downtime after the seismic event due to resulting economic and social impact (hospitals, police stations, power plants, communication centers, etc.). Nowadays, the implementation of seismic isolation (including elastomeric bearings, lead rubber bearings, sliding friction pendulum and adaptive isolation devices) and energy dissipation devices (including metallic, viscous, viscoelastic, friction, rotational and inertial dampers, tuned mass dampers and tuned liquid dampers) has become more and more common, not only for the aforementioned critical structures, but also for ordinary structures, and particularly those needing retrofitting. 
Seismic isolation and energy dissipation devices are quite mature technologies. After being studied and used for many decades, the theoretical concepts and working mechanisms underlying these systems are today well-established. Therefore, the reasons for launching a Research Topic addressing the "Recent Advances" in this field might appear questionable. Nevertheless, the use of advanced reliabilitybased or performance-based strategies capable of dealing with uncertainties inherent to the device behavior and to the response of the protected structures, are relatively modern research lines. Moreover, in recent years the design of such devices has benefitted from fast-changing improvements of numerical algorithms and optimization solvers, so that optimally configured devices implemented in real engineering projects can more easily and more accurately be obtained. Additionally, there is an ever-growing computational ability of advanced numerical models for the simulation of the mechanical behavior of these devices vis-à-vis the experimental one. Many complex non-linear phenomena significantly affecting their hysteretic behavior can be nowadays more truthfully incorporated in such models. Finally, the development of new classes of isolation devices, dissipative elements and hybrid systems for seismic control has gained momentum in recent years, encouraged by concurrent progress achieved in material science and mechanical engineering.

Following these motivations, in this Research Topic 18 high-quality papers have been selected and published following a detailed peer-review. These papers address a range of topics, including discussion of prototype tests from laboratory findings on devices available in the market, numerical studies on innovative techniques for seismic isolation or energy dissipation, case studies or benchmark projects of implemented isolation/dissipation technologies, and advanced design methodologies. In the Editors' opinion, each selected paper presents undisputable scientific novelty from various viewpoints (analytical, numerical, experimental, conceptual, implementation issues), proposes emblematic engineering projects, and represents a major contribution in the field. The Editors hope that this Research Topic can somehow contribute, even if modestly, to broadening the state of knowledge and the state of development of current and emerging mitigation strategies against the earthquake risk. An overview of the various papers gathered in this article collection is given below.

In the first paper, Pucinotti and Fiordaliso present an emblematic case study project concerning a steel-concrete bridge equipped with different energy dissipation devices, such as fluid viscous dampers and shock transmitting units integrated with the bearings. The project involves different complexities related not only to irregular altimetric and planimetric conformation, but also to the large number of devices required to guarantee the structural integrity of piers during severe design earthquakes. The proposed design procedure assisted by testing makes it possible to achieve good matching between numerical model and real dynamic response of the bridge (assessed by operational modal analysis of ambient vibrations). The proposed study represents a valid example of the most advanced structural design methods of bridges equipped with energy dissipation devices.
In the second paper, De Domenico et al. present an experimental investigation on the thermo-mechanical coupled response of friction pendulum isolators, with particular emphasis on the effect of frictional heating at the sliding interface. A series of full-scale experimental tests on a prototype isolator equipped with eight thermocouples are carried out, with an extensive testing protocol including different axial loads and sliding velocities. Temperature measurements are critically analyzed in view of the associated heat fluxes at the sliding interface. This physical phenomenon, often neglected in calculations and in building codes, produces a reduction of the energy dissipation capability of the isolator due to temperature-induced friction degradation, which in turn implies higher displacements of the isolated superstructure. The punctual temperature measurements of the eight thermocouples are useful to calibrate and validate sophisticated thermo-mechanical coupled finite element models that explicitly solve the thermal problem and the mechanical problem in an interconnected manner.

Another emblematic case study project is presented by Gandelli et al., namely the seismic retrofit of a hospital located in Southern Italy. The retrofitting intervention incorporates a series of hysteretic dissipative bracing systems. Emphasis is placed on the seismic protection of acceleration-sensitive and drift-sensitive non-structural components of the hospital, which represents a major issue due to the economic and social implications resulting from their failure. Different failure scenarios related to various intensity levels of the earthquake excitation are analyzed, in line with current performance-based design approaches. While the adopted hysteretic dampers are effective to reduce structural damage under severe design earthquakes, unacceptable peak floor accelerations during frequent design earthquakes occur, which cause serious damage to critical components like elevators and false ceilings, thus impairing the desired hospital functionality after the seismic event.

In the fourth paper, Ponzo et al. present a displacement-based design procedure for promising post-tensioned timber framed buildings coupled with hysteretic dissipative bracing systems. The numerical procedure aims at protecting both structural and non-structural elements, by identifying appropriate design force, strength, and stiffness of the post-tensioning system and of the dissipative braces. The equivalent force-displacement relation of the braced post-tensioned frame is represented by a flagshaped hysteretic behavior, which combines the equivalent bare post-tensioned frame with the equivalent elastic perfectly-plastic dissipative behavior of the bracing system. The effectiveness of the proposed system and the accuracy of the developed design procedure are verified by refined non-linear dynamic analyses and by shaking table test results carried out on $2 / 3$ scaled prototype model, and excellent agreement is found.

Effective numerical approaches for the optimal design and placement of viscous dampers in building structures accounting for uncertainties in the fault rupture slip mechanism and in the seismic input are investigated by Kondo and Takewaki, Tubaldi et al., and Navarra et al.. In particular, Kondo and Takewaki present a simultaneous treatment of the critical fault rupture slip distribution problem, via a sequential quadratic 
programming method, and the optimal damper placement problem for the critical ground motion, via a sensitivity-based method. The robustness of the maximum interstory drift in building structures under the uncertainty in fault-rupture slip distribution is analyzed by an appropriate robustness function. The analysis of the critical ground motion scenario leads to the most unfavorable structural response, so that the proposed method represents a promising tool for resilient building design. A practical design method for viscous dampers connecting adjacent structures is presented by Tubaldi et al.. The method is based on a reduced order model of the coupled system, and the typical non-linear power law force-velocity behavior of the dampers is incorporated in the design process by the stochastic linearization technique. The effectiveness of the linearized reduced order model and of the overall design strategy is assessed via numerical analyses of two adjacent buildings with shear-type behavior connected by linear or non-linear fluid viscous dampers and subjected to Gaussian stochastic base acceleration. Critical analysis and comparison of the numerical results reveals a series of useful conclusions regarding the use of linear or non-linear viscous dampers depending on the seismic intensity level, and the most convenient damper placement in relationship to the target response indicator of the adjacent structures. Along a similar research line and exploiting a similar stochastic framework, Navarra et al. develop an efficient design procedure for fluid viscous dampers implemented in building structures. The procedure aims to minimize the damper cost subjected to a constraint on the structural performance. The proposed method takes advantage of some convenient closedform expressions of the power spectral density function of the seismic input that is consistent with the pseudo-acceleration response spectrum of the installation site (expressed in a general, four-branch form valid for most of the building codes). In this way, the proposed design method is of practical connotation, as the obtained optimal design of the fluid viscous damper is coherent with the provisions of current seismic building codes.

Enhanced isolation strategies are presented by Di Matteo et al. and Zhu et al. to reduce the displacement demand of baseisolated structures and to improve the isolation performance, respectively. In particular, Di Matteo et al. comparatively investigate the performance of three unconventional hybrid isolation strategies, consisting in the combination of the base isolation (BI) with: (1) a tuned mass damper (TMD) on the basement of the structure; (2) a new TMD with a dashpot located in between the secondary mass and the ground; (3) a tuned liquid column damper (TLCD). The performance is analyzed in terms of base displacement, acceleration, and stroke of the devices. It is found that the new TMD is more effective that the other two analyzed passive devices due to the higher dissipative forces, and can represent an effective system to reduce the displacement response of baseisolated structures. Zhu et al. propose a semi-active non-linear damping-based building isolation system under seismic loadings. A two-degree-of-freedom (2-DOF) scaled building model is developed for simulation studies. Calibrated and validated against experimental results, the 2-DOF model is then used to carry out the optimal design of the non-linear damping parameter to minimize the acceleration and the inter-story drift of the building.

Another emblematic case study project concerning a seismic retrofitting intervention with hysteretic damped braces is presented by Mazza and Imbrogno. The peculiarity of this study is that the authors analyze a reinforced concrete (RC) school in Southern Italy under a critical scenario in which such structure is damaged by a fire. Different extents of damage are related to different durations of heating and cooling phases, and two fire scenarios (related to the extension of the fire compartment) are studied. A displacement-based design procedure of the hysteretic dampers is adopted to obtain a retrofitted structure globally regular with regard to stiffness and strength, by balancing the degradation of fire-exposed RC frame members. Therefore, the proposed procedure fully accounts for the damaged properties of the RC cross sections due to fire, and represents a valuable numerical tool to account for concurrent risk scenarios (earthquake and fire) when designing retrofitting interventions.

A comprehensive computer-aided seismic design tool for both new and existing structures equipped with hysteretic dampers is presented by Nuzzo et al.. This computational tool, named DIBRAST (design of Dissipative BRAced Structures), underlies a specific displacement-based design procedure developed by the same authors. Unlike other articulated procedures that require iterative steps at the design stage, the proposed methodology is implemented in a closed-form analytical variant wherein the required iterations are automated by the software, thus significantly reducing computational times. The code calculates the mechanical properties of the dissipative system, in terms of yielding force and elastic stiffness, which are able to meet the target performance objective in terms of displacement. The codes proves to be a promising computational tool aiming at simplifying the (sometimes intricate) design procedures of structures equipped with hysteretic dampers.

An innovative structural control system for high-rise buildings under pulse-type and long-duration earthquake ground motions is developed by Kawai et al.. In particular, a damping layer is inserted between two stiff cores, a sub-frame upper strong-back core and a sub-frame lower strong-back core, so that the deformation is concentrated in the connection point of the cores. The behavior of the proposed damping configuration is investigated by analyzing the resulting mode shapes with different damping levels. Then, a simplified model is proposed to allow a direct investigation of the control performance. The comparison between the simplified and full models is also provided. Finally, different types of inputs (i.e., transient-like inputs and seismic excitation) are considered to evaluate the control effectiveness. The optimal damping coefficient allows to achieve quite good response reductions when subjected to far field, near-fault pulse-like, and long-period earthquakes.

In the paper by Furinghetti et al., a hybrid simulation technique is implemented in order to assess the effectiveness of a curved surface slider in reducing the seismic response of a base isolated building. In particular, while the dynamic behavior of the superstructure is investigated by a numerical model, an isolation device (representative of the whole set of isolators) is physically 
tested and both outcomes are considered together in a hybrid testing framework. The response from the hybrid simulation is compared with the response obtained with a full numerical building model in which the non-linear isolator cyclic response is implemented, and very good agreement is found. The proposed hybrid simulation technique is able to overcome the difficulties that an experimental test of a full-scale base isolated building could entail and, at the same time, allows capturing the essential dynamical characteristics of the system under investigation.

Another emblematic case study project is presented by Dall'Asta et al.. In particular, this paper presents the seismic design of the new Camerino University Research Center (Italy). The building consists of a steel braced superstructure with pinned joints and RC sub-structures able to fit the complex morphology of the installation area. In order to enhance the seismic performance of the building under different earthquake intensity levels concerning both serviceability and ultimate limit states, a hybrid base-isolation system consisting of high-damping rubber bearings and low-friction flat sliders, with a high period of isolation, is designed. A resilience and robustness oriented design procedure, based on a probabilistic seismic hazard analysis for the building site, is developed. The seismic performance is analyzed by a specific risk analysis and results are presented in terms of hazard curves for different return periods of the earthquake excitation relevant to both the isolation system and the superstructure. The results demonstrate a high level of safety and robustness of the building as well as a high level of resilience, thus making the proposed case study a representative example of earthquake-resilient building design.

Scale effects are of paramount importance in experimental tests to investigate the hysteretic behavior of isolation devices. Using scaled devices would allow cheaper and more feasible characterization, but the validity of the obtained results against the real-scale isolator may be questionable. The interesting contribution by Murota and Mori deals with such scale effects on a high damping rubber bearing. In particular, experimental tests are carried out on two types of isolators: full-scale isolators (with $1,000 \mathrm{~mm}$ diameter) requiring large capacity testing equipment, and scaled isolators (with $225 \mathrm{~mm}$ diameter) requiring smaller testing equipment and implying reduced costs. Shear-strain dependence tests and frequency dependence tests are performed on both the isolators; then, repeated loading tests are performed on the scaled model isolator. Finite element analysis is also carried out to simulate the repeated loading test on isolators with larger dimensions. Results show that, although the absolute values of the equivalent shear properties of the two bearings are different, the normalized variation trends as a function of the frequency and of the strain amplitude are relatively similar. Furthermore, repeated loading tests show that the history dependence and temperature dependence effects are remarkable and lead to a progressive loss of the shear stiffness and dissipation capacity of the bearing. Finally, the finite element analysis demonstrates that the loss rate with increasing number of cycles depends on the internal temperature increment and, hence, is strictly related to the isolator dimensions.

Ground motions with velocity pulses caused by nearfault directivity have received a great deal of attention from engineers and seismologists because of their potential to cause severe damage to structures, especially for flexible structures. Three papers of the Research Topic by Anajafi et al., Elias et al., and Hashizume and Takewaki specifically address this aspect. In particular, in the paper by Anajafi et al., the effectiveness of base isolation for long-period structures is assessed. A preliminary numerical study on inelastic displacement spectra is presented to investigate the effectiveness of seismic isolation for flexible structures. Despite the common belief that this solution is detrimental for such structures, some beneficial effects are highlighted in this study, for both far-fault (FF) and near-fault (NF) seismic events. Then, an emblematic case study project concerning the Rudshur Bridge (Iran) is analyzed. This bridge is a composite steel box girder bridge with long piers and long periods, placed in an area prone to NF excitations but designed for $\mathrm{FF}$ earthquakes. It is found that the seismic isolation can be effective even for this long-period bridge under both FF and NF excitation, with a reduction of both base shear and pier drift. Since the deck may undergo large displacements under NF (the larger the strength reduction, the larger the deck displacements), an optimum value of yield strength-toweight ratio is identified. From another perspective, the use of TMD schemes is commonly discouraged for structures subjected to short-duration, pulse-like ground motions such as the aforementioned near-field earthquake excitations. While the efficiency of TMDs on structures under far-field earthquakes has been demonstrated, the convenience of its employment against near-fault earthquakes is still under discussion. The paper by Elias et al. investigates the response mitigation of a reinforced concrete chimney subjected to pulse-like nearfault ground motions with different TMD schemes, namely single TMD (SMTD), multiple TMD having equal stiffness (wMTMDs) and multiple TMD having equal masses (e-MTMTs). Based on time-history analyses under 69 pulse-like ground motions, it is found that the pulse period of ground motion plays a very important role in how effective the TMD control schemes are. In particular, the e-MTMDs schemes prove to be effective in reducing the response of the chimney while subjected to earthquakes having dominant periods close to higher order periods, whereas the STMD is quite effective for controlling the fundamental period. Finally, in the paper by Hashizume and Takewaki, a new viscous-hysteretic hybrid (HVH) damping system is proposed for seismic control against long-period pulse-type earthquake ground motions of large amplitude. The proposed system includes a viscous damper and a hysteretic damper with a gap mechanism in parallel. In the proposed damper system, the viscous damper allows controlling the broad-amplitude range vibration whereas the hysteretic damper with a gap mechanism is expected to act as a stopper for large-amplitude range vibration. The proposed $\mathrm{HVH}$ damper system is a promising control system for reducing the response of long-period structures (e.g., high-rise buildings and base-isolated buildings) under pulse-type ground motions of large amplitude, without implying large residual deformation (which is an advantage compared to alternative dual hysteretic damper systems). 


\section{AUTHOR CONTRIBUTIONS}

All authors listed have made a substantial, direct and intellectual contribution to the work, and approved it for publication.

\section{ACKNOWLEDGMENTS}

We would like to thank all authors for their valuable contributions, the many dedicated reviewers for their useful guidance to improve the papers, and the Editorial Team of Frontiers in Built Environment (Earthquake Engineering Section) for the professional assistance and for the valuable opportunity to launch this Research Topic.

Conflict of Interest: The authors declare that the research was conducted in the absence of any commercial or financial relationships that could be construed as a potential conflict of interest.

Copyright (c) 2020 De Domenico, Tubaldi, Takewaki, Karavasilis, Dall'Asta and Lavan. This is an open-access article distributed under the terms of the Creative Commons Attribution License (CC BY). The use, distribution or reproduction in other forums is permitted, provided the original author(s) and the copyright owner(s) are credited and that the original publication in this journal is cited, in accordance with accepted academic practice. No use, distribution or reproduction is permitted which does not comply with these terms. 